The Role of Maternal Cardiac Vagal Control in the Association between Depressive Symptoms and Gestational Hypertension

Codie R. ROULEAU ${ }^{\mathrm{a}}$, Lianne M. TOMFOHR-MADSEN ${ }^{\mathrm{a}}$, Tavis S. CAMPBELL ${ }^{\mathrm{a}}$, Nicole LETOURNEAU $^{\mathrm{b}, \mathrm{c}}$, Maeve O’BEIRNE ${ }^{\mathrm{d}}$, Gerald F. GIESBRECHT ${ }^{\mathrm{a}, \mathrm{b}, \mathrm{c}, *}$ \& The APrON Study Team

${ }^{\text {a} D e p a r t m e n t ~ o f ~ P s y c h o l o g y, ~ U n i v e r s i t y ~ o f ~ C a l g a r y ~}$ 2500 University Drive NW, Calgary, AB, Canada T2N 1N4

${ }^{\text {b} A l b e r t a ~ C h i l d r e n ' s ~ H o s p i t a l ~ R e s e a r c h ~ I n s t i t u t e ~ f o r ~ C h i l d ~ a n d ~ M a t e r n a l ~ H e a l t h ~}$ 3330 Hospital Drive NW, Calgary, AB, Canada, T2N 4N1

'Department of Paediatrics, University of Calgary 2888 Shaganappi Trail NW, Calgary, AB, Canada, T3B 6A8

${ }^{\mathrm{d}}$ Department of Family Medicine, University of Calgary 2500 University Drive NW, Calgary, AB, Canada T2N 1N4

*Corresponding author. Office: +1 (403) 441-8469, Fax: +1 (403) 210-9529 Child Development Centre, Room 355, 3820-24 Avenue NW Calgary, Alberta Canada T3B 2X9 Email address: ggiesbre@ucalgary.ca

\title{
(cc) EY-NC-ND
}

(C) 2016. This manuscript version is made available under the CC-BY-NC-ND 4.0 license https://creativecommons.org/licenses/by-nc-nd/4.0/ 


\begin{abstract}
Reduced cardiac vagal control, indexed by relatively lower high-frequency heart rate variability (HF-HRV), is implicated in depressed mood and hypertensive disorders among non-pregnant adults whereas research in pregnancy is limited. This study examined whether maternal HF-HRV during pregnancy mediates the association between depressed mood and gestational hypertension. Depressive symptoms (Edinburgh Depression Scale) and HF-HRV were measured during early $(M=14.9$ weeks $)$ and late $(M=32.4$ weeks $)$ pregnancy in 287 women. Gestational hypertension was determined by chart review. Depressive symptoms were associated with less HF-HRV $(b=-0.02, p=.001)$. There was an indirect effect of depressed mood on gestational hypertension through late pregnancy HF-HRV $(b=0.04,95 \%$ CI $0.0038,0.1028)$ after accounting for heart rate. These findings suggest cardiac vagal control is a possible pathway through which prenatal depressed mood is associated with gestational hypertension, though causal ordering remains uncertain.
\end{abstract}

Keywords: cardiac vagal control, high-frequency heart rate variability, heart rate, depressive symptoms, gestational hypertension, pregnancy 


\section{Highlights}

- Depressed mood relates to less high-frequency heart rate variability (HF-HRV) in pregnancy.

- Less maternal HF-HRV is associated with gestational hypertension.

- Depressed mood shows an indirect effect on gestational hypertension via HF-HRV. 


\section{Introduction}

The past decade has seen a growing interest in potential impacts of psychosocial distress during pregnancy on maternal and child health outcomes (Dunkel-Schetter \& Tanner, 2012; Staneva, Bogossian, Pritchard, \& Wittkowski, 2015). Within this context, depressed mood is among the most widely studied and common types of prenatal distress, with $9-22 \%$ of pregnant women reporting significant depressive symptoms (Lee et al., 2007; Shakeel et al., 2015). It has been shown that prenatal depressive symptoms are associated with obstetric complications (Alder, Fink, Bitzer, Hösli, \& Holzgreve, 2007; Dunkel-Schetter, 2011), such as gestational hypertension (Cripe, Frederick, Qiu, \& Williams, 2011; Leeners, Neumaier-Wagner, Kuse, Stiller, \& Rath, 2007; Zhang et al., 2013).

Gestational hypertension is characterized by elevated maternal blood pressure with onset after 20 weeks gestation (Heazell, Norwitz, Kenny, Louise, \& Baker, 2010; Magee et al., 2014), and affects $6-17 \%$ of nulliparous and $2-4 \%$ of multiparous pregnancies (Heazell et al., 2010; Sibai, 2003). Although gestational hypertension does not typically lead to adverse perinatal outcomes, it can be a symptom of preeclampsia which can further progress into severe complications including haemolysis elevated liver enzymes low platelets counts (HELLP) syndrome as well as maternal and fetal morbidity and mortality (Heazell et al., 2010; Sibai, 2003). A meta-analysis of 13 case-control and cohort studies revealed that a composite measure of prenatal depressive symptoms, anxiety, pregnancy-related stress, and work stress was associated with 1.3- to 1.5-fold increases in risk of gestational hypertension and preeclampsia, respectively (Zhang et al., 2013). Mechanistic studies in non-pregnant adults suggest depression impacts cardiovascular health through behavioral and biological mechanisms (Miller, Chen, \& 
Cole, 2009; Whooley \& Wong, 2013), but the pathways through which prenatal depressed mood is associated with gestational hypertension are currently unclear.

\section{Cardiac Vagal Control, Blood Pressure Regulation, and Gestational Hypertension}

A little investigated but plausible mechanism linking depressed mood to gestational hypertension is cardiac vagal control (Pal et al., 2011; Shea et al., 2008) - i.e., the input to the heart from the parasympathetic branch of the autonomic nervous system. The autonomic nervous system plays a prominent role in regulating cardiac output and vascular resistance, the two main determinants of blood pressure (Beevers, Lip, \& O’Brien, 2001; Guyenet, 2006; Liao et al., 1996). Cardiac output is determined by end-diastolic volume, myocardial contractility, and heart rate (Guyenet, 2006). With respect to heart rate, pressure-sensitive cells embedded in arterial walls convey information about blood flow to the nucleus of the solitary tract by afferents in the vagal and glossopharyngeal nerves (Thayer, Hansen, \& Johnsen, 2008). In response to changes in blood flow, outgoing autonomic activity is adjusted such that acute reductions in cardiac vagal activity and/or increases in sympathetic activity lead to transitory increases in heart rate, vascular resistance, cardiac output, and blood pressure (Purves et al., 2001). If sustained, these increases in blood pressure can contribute to the development of hypertension (Beevers et al., 2001).

Consistent with its vital role in blood pressure regulation, there is evidence that cardiovascular autonomic control may contribute to gestational hypertension. Pregnancy is commonly regarded as a "vascular stress test," given the tremendous cardiovascular adaptations that are required for a healthy pregnancy (Heazell et al., 2010). With advancing gestation, these adaptations include increased heart rate, increased cardiac output, increased blood volume, reduced heart rate variability (HRV), and attenuated cardiovascular reactivity (de Weerth \& Buitelaar, 2005; Klinkenberg et al., 2009; Stein et al., 1999). Although the exact etiology is 
unclear, gestational hypertension is thought to represent failure to adapt to these cardiovascular changes, triggered by a multitude of factors including genetic, immunological, and environmental influences, and abnormal placentation (Heazell et al., 2010). In gestational hypertension, this failure to adapt is manifest as elevated heart rate and cardiac output, with normal vascular resistance, relative to healthy pregnancy (Bosio, McKenna, Conroy, \& O’Herlihy, 1999; De Paco, Kametas, Rencoret, Strobl, \& Nicolaides, 2008; Pal et al., 2011). In particular, longitudinal data show that cardiac output increases at a greater rate in gestational hypertension compared to in healthy pregnancy (Bosio et al., 1999). Much attention has been paid to excessive sympathetic activity as a contributor to increases in heart rate and cardiac output that are observed in gestational hypertension (Greenwood, Scott, Walker, Stoker, \& Mary, 2003; Rang, Wolf, Montfrans, \& Karemaker, 2002), whereas the other component of autonomic blood pressure regulation — namely, cardiac vagal control—-has not been widely assessed in this context.

Cardiac vagal control can be measured indirectly via HRV, the beat-to-beat variation of time intervals between consecutive heartbeats (Thayer et al., 2008). Relatively lower HRV (i.e., less beat-to-beat variation) is associated with cardiovascular risk factors and disease in nonpregnant adults (Thayer, Yamamoto, \& Brosschot, 2010), including hypertension (Schroeder et al., 2003; Thayer et al., 2010). In particular, the high frequency (HF; 0.15-0.4 Hz) component of HRV has been used as an estimate of cardiac vagal activity (Berntson et al., 1997; Pomeranz et al., 1985). Whereas heart rate is driven by a combination of sympathetic and parasympathetic activity (Berntson, Cacioppo, \& Quigley, 1991), HF-HRV is influenced more selectively by the parasympathetic branch of the autonomic nervous system. Reductions in HF-HRV are therefore thought to reflect reduced parasympathetic activation of the heart, which correspond to decreases 
in R-R interval length (i.e., higher heart rate) under conditions of selective parasympathetic influences on the heart (however, see Grossman \& Taylor, 2007, for sizeable respiratory influences on HF-HRV). Measurement of HF-HRV during pregnancy complements existing knowledge about the autonomic nervous system influences in gestational hypertension by clarifying the role of cardiac vagal control.

\section{Depressive Symptoms and Cardiac Vagal Control}

In non-pregnant adults, it has been repeatedly demonstrated that lower HRV is associated with various forms of psychosocial distress, including depression and anxiety (Chalmers, Quintana, Abbott, \& Kemp, 2014; Friedman \& Thayer, 1998; Horsten et al., 1999; Rottenberg, 2007), episodes of worry (Pieper, Brosschot, van der Leeden, \& Thayer, 2007; Thayer, Friedman, \& Borkovec, 1996), and work stress (Kang et al., 2004). In the short-term, increases in heart rate that result from reduced parasympathetic and/or increased sympathetic activation reflect an adaptive response to psychosocial distress, allowing a quick reaction to perceived threat. In response to longer term psychosocial distress, however, persistent autonomic imbalance can lead to excessive wear-and-tear on physiological systems that support blood pressure regulation and can contribute to increasingly high resting blood pressure (McEwen \& Seeman, 2006; Thayer \& Lane, 2007). Consistent with these findings, depressed mood is related to hypertension in non-pregnant adults and there is evidence that the relationship is at least partially mediated by cardiac vagal control (Carney, Freedland, Miller, \& Jaffe, 2002; Meng, Chen, Yang, Zheng, \& Hui, 2012).

Little is known about the association between mood and HRV in pregnancy, perhaps in part because these associations may be obscured by the complex changes in cardiac activity that occur during healthy pregnancy (de Weerth \& Buitelaar, 2005). Shea and colleagues (2008) 
report that pregnant women (mean gestational age $=27.2$ weeks) with clinically significant depressive symptoms had lower 24-hour ambulatory HRV and higher heart rate than women without significant depressed mood. This finding suggests that depressed mood may be associated with lower maternal HRV, but more work is needed to understand how HRV relates to adverse pregnancy outcomes such as gestational hypertension.

\section{The Present Study}

Elevated depressive symptoms are relatively common in pregnancy and are known to correlate with autonomic mechanisms involved in blood pressure regulation, including changes in heart rate and cardiac output. However, little attention has been paid to the potential impact of depressive symptoms on gestational hypertension via pathophysiological changes in cardiac vagal control. Our first aim was to replicate prior findings showing that depressive symptoms are associated with relatively higher heart rate and lower HF-HRV in pregnancy (Shea et al., 2008), using a longitudinal design and appropriate covariates. Our second aim was to investigate whether cardiac vagal control mediates the relationship between depressed mood and gestational hypertension, over and above the influence of heart rate. We hypothesized that (1) depressive symptoms would be associated with higher maternal heart rate and lower HF-HRV during pregnancy, and (2) maternal HF-HRV would mediate the association between depressed mood and increased risk for gestational hypertension.

\section{Methods}

\section{Participants}

Participants included a community sample of pregnant women who were recruited to participate in the Fetal Programming Study, a longitudinal cohort study designed to assess the effects of prenatal stress on maternal health and infant development. Exclusion criteria for the 
current analysis included: (a) $>22$ weeks gestation at baseline, (b) cardiovascular disease or hypertension (blood pressure $>140 / 90 \mathrm{mmHg}$ ) prior to pregnancy or at the time of enrolment, (c) non-singleton pregnancy, (d) self-reported use of cigarettes, illicit drugs, or alcohol during the current pregnancy (as these are known to impact cardiac vagal tone and/or blood pressure; Fuchs, Chambless, Whelton, Nieto, \& Heiss, 2001; Thayer et al., 2010), (e) illness during the two-day data collection period (e.g., fever, influenza) that may have impacted study variables or adherence to the study protocol, and (f) current use of medications that influence cardiovascular fluctuations including anti-hypertensive medications (e.g., beta-blockers, calcium channel blockers). All participants provided written informed consent. Procedures for the present study were approved by the University of Calgary Conjoint Health Research Ethics Board.

\section{Materials and Procedure}

The assessment schedule for study variables is included in Figure 1. Data collection was completed in early pregnancy $(M=14.9$ weeks, $S D=3.6)$ and late pregnancy $(M=32.4$ weeks, $S D=1.0$ ). During each data collection period, heart rate and physical activity were continuously recorded for two consecutive days (excluding weekends) using ambulatory assessment.

Participants also completed questionnaires assessing depressed mood and anxiety in early and late pregnancy. An individualized training session showed participants how to operate data collection devices including a heart rate monitor (for measurement of HF-HRV), actigraph watch (for measurement of objective physical activity), and personal digital assistant (for measurement of self-reported exercise and napping). Recordings from the personal digital assistant, heart rate monitor, and actigraph were electronically time-stamped to enable comparisons across measures. Demographics, obstetric and medical history (including absence of pre-existing hypertension), gestational age, and information about prescribed medications (including antidepressants and 
anti-hypertensives) were obtained at the time of study enrollment through a self-report questionnaire, and were confirmed by review of medical charts following delivery. As described below, gestational hypertension was diagnosed after 20 weeks gestation, and was determined by chart review following delivery.

\section{Measures}

Prenatal depressive symptoms. Depressive symptoms were assessed using the Edinburgh Depression Scale (EDS) (also known as the Edinburgh Postnatal Depression Scale; Cox, Holden, \& Sagovsky, 1987), a 10-item instrument widely used to screen for perinatal depression. Items are rated on a four-point scale and summed to provide a total score ranging from 0 to 30, with higher scores representing more severe depressed mood. Scores $>12$ are predictive of a major depressive disorder (Cox et al., 1987) and a change of \pm 4 points is considered clinically meaningful (Matthey, 2004). The EDS has excellent reliability and validity among pregnant women (Nast, Bolten, Meinlschmidt, \& Hellhammer, 2013).

Ambulatory assessment of heart rate and HF-HRV. HF-HRV was measured in order to specifically examine parasympathetic influences on the heart (Thayer et al., 2008). R-R intervals were recorded using the Firstbeat Bodyguard (Firstbeat Technologies OyTM, Jyvaskyla, Finland), a small ambulatory monitor attached to two electrodes (3M, London, Canada). Three five-minute segments were sampled from a 24-hour sequence of R-R intervals at the following times: 30 minutes after waking, at 11:00, and at 21:00. These times were chosen to correspond with self-report measures of physical activity. This sampling paradigm resulted in 12 samples of heart rate and 12 samples of HF-HRV for each participant (six in early pregnancy, six in late pregnancy). Five minutes is a standard interval for short-term HRV recordings (Kreibig, 2010; Task Force, 1996). Each five-minute segment was visually inspected for artifacts or 
physiologically improbable RR intervals (Berntson, Quigley, Jang, \& Boysen, 1990). Artifactual data were replaced by interpolating missing R-waves from surrounding beats (Berntson et al., 1990), using Nevrokard $a$ HRV software (Medistar, Izola, Slovenia). HF-HRV was calculated using power spectral analysis with Fast Fourier transformation. HF-HRV values were logtransformed due to a skewed distribution, and are expressed as $\ln \left(\mathrm{ms}^{2}\right)$. Heart rate is expressed in beats per minute (bpm).

Gestational hypertension. Gestational hypertension is defined as systolic blood pressure $\geq 140 \mathrm{mmHg}$ and/or diastolic blood pressure $\geq 90 \mathrm{mmHg}$ that develops for the first time after 20 weeks gestation (Magee et al., 2014). Blood pressure was manually recorded during routine obstetrician/physician physical exams, beginning in early pregnancy. Gestational hypertension was diagnosed in a dichotomous manner (i.e., present vs. absent) by the physician and documented in the health records. The presence of gestational hypertension was determined by chart review following delivery.

Physical activity. Physical movement is a key determinant of daytime fluctuations in heart rate (Bernardi, Valle, Coco, Calciati, \& Sleight, 1996), highlighting the importance of accounting for concurrent physical activity in ambulatory HRV studies. We measured momentary physical activity in two ways. First, participants were prompted to report on the personal digital assistant if they had exercised vigorously (e.g., running) or slept in the 30 minutes preceding each time point corresponding to HRV sampling; segments of cardiac activity were excluded from analysis if the participant responded "yes" to either question. Second, physical activity was continuously recorded during the two-day data collection period using a wrist actigraph (Ambulatory Monitoring, Ardsley, USA) worn on the non-dominant hand. Wrist actigraphy has demonstrated correlations with oxygen uptake $(r=.73)$, heart rate $(r=.71)$ and 
self-reported physical activity $(r=0.53-0.81)$, and can differentiate between periods of sedentary versus physical activity (Patterson et al., 1993). Actigraphs were initialized using one-minute epoch intervals and the proportional integration mode that integrates area under the accelerometer waveform curve to index average vigor of motion (Blackwell et al., 2008; Garcia, Langenthal, Angulo-Barroso, \& Gross, 2004). Physical activity was calculated using a validated scoring algorithm (Jean-Louis, Kripke, Mason, Elliott, \& Youngstedt, 2001) in Action-W 2.7.1 software (Ambulatory Monitoring, Ardsley, USA) for each five-minute time segment corresponding to HF-HRV measures. Off-wrist periods were detected by Action-W and excluded from analysis. Each time segment yielded a mean activity score, measured in counts per epoch. Actigraphy was also used to measure average physical activity over the sampling days by calculating a mean activity score for all waking hours during the two-day data collection periods in early and late pregnancy.

Pre-pregnancy body mass index. Pre-pregnancy body mass index (BMI) was derived by self-reported weight prior to becoming pregnant and measurement of height by a trained research assistant during the early pregnancy assessment.

Prenatal anxiety. Anxiety was evaluated in order to examine depressed mood independent of anxiety, given their common features (e.g., negative affect) and frequent cooccurrence (Clark, Watson, \& Mineka, 1994). We measured pregnancy anxiety using a 10-item questionnaire that evaluates worry about pregnancy issues such as maternal and infant health, labor, delivery, and infant care (Rini, Dunkel-Schetter, Wadhwa, \& Sandman, 1999). Items are rated on a four-point scale and averaged to provide a total score ranging from 1 to 4 with higher scores indicating greater pregnancy anxiety (Rini et al., 1999). This measure has demonstrated 
good reliability (Cronbach's $\alpha=0.81$ ) (Rini, Dunkel-Schetter, Hobel, Glynn, \& Sandman, 2006) and concurrent validity with state anxiety (Rini et al., 1999).

\section{Data Analysis}

Hypothesis 1. Hierarchical linear modeling (HLM) was used to test if depressive symptoms were related to HF-HRV and heart rate assessed in early and late pregnancy. HLM was selected because it is well-suited to handle nested and missing data, and does not require an equal number of observations across individuals. Using an unstructured covariance structure, multilevel equations were specified at three levels, with measurement moments (Level 1) nested within days (Level 2) nested within persons (Level 3). Two separate models evaluated the relationship between depressive symptoms and HF-HRV (Model 1) and heart rate (Model 2), after adjusting for covariates determined a priori including pre-pregnancy BMI, maternal age, momentary physical activity, and gestational age (weeks). The following equations served as the basis for Model 1:

Level 1: HF-HRV ${ }_{t i j}=\pi_{0 i j}+\pi_{l i j} *$ Activity $\left._{t i j}\right)+e_{t i j}$

Level 2: $\pi_{0 i j}=\beta_{00 j}+\beta_{01 j} *\left(\mathrm{GA}_{i j}\right)+r_{0 i j}$

Level 3: $\beta_{00 j}=\gamma_{000}+\gamma_{001}\left(\mathrm{EDS}_{j}\right)+\gamma_{002}\left(\mathrm{BMI}_{j}\right)+\gamma_{003}\left(\mathrm{Age}_{j}\right)+u_{00 j}$

where HF-HRV represents log-transformed HF-HRV and Activity represents mean-centered momentary activity scores, measured concurrently at time segment $t$, on day $i$, and within person $j$. GA in the Level 2 model refers to gestational age at the time of assessment on day $i$ within person $j$. Individual-level variables in Level 3 refer to depressive symptoms in early pregnancy, pre-pregnancy BMI, and maternal age for person $j$. Model 2 was identical except that heart rate was included as the outcome variable instead of HF-HRV. Data were analyzed with HLM 7.0 software (Scientific Software International, Skokie, USA). The regression coefficient relating to 
depressive symptoms was tested for statistical significance $(p<0.05)$ and interpreted as the number of units change in log HF-HRV (Model 1) or heart rate (Model 2) associated with a oneunit change on the EDS. In order to rule out the potential influence of other confounds, we also ran the models with prenatal anxiety, average physical activity, parity, and dichotomized measures of education and family income as covariates.

Hypothesis 2. Mediation analyses were conducted using the product of coefficient approach implemented by the PROCESS macro (Hayes, 2013) in SPSS 21.0 (IBM Corporation, Armonk, USA) to test whether there were indirect effects of depressed mood on gestational hypertension via HF-HRV, while adjusting for maternal age, pre-pregnancy BMI, and momentary activity. We used a parallel mediation model with both HF-HRV and heart rate as proposed mediators (see Figure 2). The parallel mediation approach allowed us to evaluate the association between depressive symptoms and gestational hypertension via changes in HF-HRV (which is primarily associated with vagal activity), incremental to potential mediating influences of heart rate (which is associated with a mixture of vagal and sympathetic activity; Thayer et al., 2008).

We separately evaluated early pregnancy versus late pregnancy cardiac activity. To do so, we ran the parallel mediation model with early pregnancy (Figure 2, Panel I) and late pregnancy (Figure 2, Panel II) HF-HRV and heart rate as simultaneous mediators. This approach allowed us to examine whether changes in HF-HRV and/or heart rate could be detected prior to the development of gestational hypertension. The early pregnancy assessment, which always occurred at less than 22 weeks gestation, helped ensure cardiac activity was measured before the development of gestational hypertension (by definition, diagnosed after 20 weeks gestation). The dependent variable in all four models was gestational hypertension and the independent variable 
was early pregnancy EDS. For the mediation analyses, heart rate and HF-HRV were computed as the average of all samples within each time point.

Note that we report results in terms of direct and indirect effects to be consistent with conventional terminology for mediation analysis (Hayes, 2013), while acknowledging that causality cannot be clearly established given our research design. We report bias-corrected bootstrap confidence intervals (95\%) around the indirect effect based on 5,000 re-samples. A significant indirect effect exists if the confidence interval surrounding the point estimate for the $a b$ path does not include 0 (Efron \& Tibshirani, 1993; Hayes, 2013). Gestational hypertension was coded dichotomously $(1=$ present, $0=$ absent $)$; therefore, coefficients from analyses wherein gestational hypertension is the dependent variable result from logistic regression and denote the amount of change expected in the log odds of gestational hypertension when there is a one-unit change in the predictor (Meyers, Gamst, \& Guarino, 2013). To facilitate interpretation of logistic regression results, point estimates and confidence interval endpoints were exponentiated $\left(e^{\log \text { odds }}\right)$ to translate them to an odds ratio metric (Hosmer, Lemeshow, \& Sturdivant, 2013). We also ran the mediation models with other potential covariates (prenatal anxiety, average physical activity, parity, education, income) to further rule out confounding influences.

\section{Results}

\section{Descriptive Statistics}

Overall, 294 women enrolled in the study and completed an early pregnancy assessment. Seven were excluded due to pre-existing cardiovascular disease $(n=2)$, use of medications that influence cardiovascular fluctuations ( $n=3$; all beta-blockers), difficulty understanding the study instructions $(n=1)$, or not providing any data relevant to the current analysis $(n=1)$, yielding a final sample of 287 participants. Of these, 253 completed a late pregnancy assessment. Missing 
the late pregnancy assessment was not associated with gestational hypertension, maternal age, BMI, early pregnancy depressive symptoms, HF-HRV, gestational age, or actigraphy scores (all correlations $p>.05)$. We were able to obtain valid HRV data from 271 participants $(94.4 \%)$ in early pregnancy and $242(95.7 \%)$ in late pregnancy. Of the total possible 3240 samples of momentary HRV, missing data were due to self-reported sleep/exercise during the sampling moment $(n=58,1.8 \%)$, non-response to the personal digital assistant - which prevented us from confirming whether they had exercised or napped during that HRV sampling period $(n=116$, $3.6 \%)$, excessive artifacts $(n=282,8.7 \%)$, equipment failure $(n=47,1.4 \%)$, and participants not wearing the heart monitoring device $(n=243,7.5 \%)$. Altogether, 271 participants $(94.4 \%)$ in early pregnancy and $242(95.7 \%)$ in late pregnancy provided at least one HRV sample. Most women provided all six scheduled HRV-Activity samples during a given data collection period, while the average number of missing samples was 0.8. All remaining variables (e.g., EDS, demographics, gestational hypertension) had less than 5\% missing data. For the HLM analysis, missing data were dealt with using full information maximum likelihood. For the mediation analyses, missing data were imputed using the expectation maximization algorithm available in SPSS. This approach was deemed appropriate based on Little's MCAR test of variables included in the mediation models, which was not significant $\left(\chi^{2}=70.79, d f=70.79, p=.965\right)$, indicating there was no evidence to suggest the data were other than missing completely at random.

Sample characteristics are reported in Table 1, as a function of gestational hypertension. Incidence of gestational hypertension was $6.6 \%$. Participants ranged in age from 19 to 43 years $(M=31.4, S D=3.8)$, were married or in common law relationships $(n=273,95.1 \%)$, had completed more than high school education $(n=247,86.1 \%)$, and half of the sample reported household incomes of $\$ 100,000$ Canadian or more $(n=151,52.6 \%)$. There was a wide range of 
pre-pregnancy BMI values from underweight $\left(<18.5 \mathrm{~kg} / \mathrm{m}^{2} ; n=9,3.1 \%\right)$ to obese $\left(\geq 30 \mathrm{~kg} / \mathrm{m}^{2} ; n\right.$ $=43,15.0 \%)$, though mean BMI $\left(M=25.0 \mathrm{~kg} / \mathrm{m}^{2}, S D=5.2\right)$ fell in the normal range. Sixty-two percent $(n=178)$ of women reported having been pregnant before. HF-HRV was inversely correlated with heart rate in both early pregnancy $(r=-.62, p<.001)$ and late pregnancy $(r=.-$ $.61, p<.001)$

Average EDS at both time points fell below the cutoff for clinically significant depressive symptoms $(>12)$. There was no significant change in EDS scores from early $(M=5.7, S D=4.2)$ to late $(M=5.3, S D=4.1)$ pregnancy, $t(253)=1.63, p=.104$. Less than $5 \%$ of women $(n=14)$ showed clinically significant change across EDS diagnostic categories. Because these findings suggest that depressive symptoms were stable across pregnancy, and to ensure our measure of depressive symptoms preceded the onset of gestational hypertension, all of our hypotheses were tested using only early pregnancy depressive symptoms.

\section{Associations between Depressive Symptoms, HF-HRV, and Heart Rate}

Table 2 presents results from two hierarchical linear models evaluating the association between depressive symptoms in early pregnancy and HF-HRV (Model 1) and heart rate (Model 2). After accounting for maternal age, pre-pregnancy BMI, momentary activity, and gestational age, HF-HRV was negatively associated with depressive symptoms $(b=-0.02, S E=.005, t(267)$ $=-3.26, p=.001)$. Similarly, Model 2 shows that higher heart rate was related to worse depressive symptoms $(b=0.44, S E=.115, t(267)=3.81, p<.001)$. The addition of anxiety, average physical activity, parity, education, and income to either model did not change the findings. We also re-ran both models excluding women with documented antidepressant use during pregnancy $(n=17)$; the significant coefficient for EDS remained unchanged. 


\section{Indirect Effects of Depressive Symptoms on Gestational Hypertension through HF-HRV}

The indirect effects of HF-HRV and heart rate in the association between early pregnancy depressive symptoms and gestational hypertension are presented in Figure 2 (see full details in Table S3). The model for early pregnancy HF-HRV and heart rate (Panel I, Figure 2) showed no indirect effects of early pregnancy depressive symptoms on gestational hypertension via either HF-HRV or heart rate $(b=0.01, S E=.014 ; 95 \%$ CI $-0.0053,0.0540$ and $b=0.02, S E=.021$; 95\% CI -0.0164, 0.0666, respectively). In contrast, the model assessing late pregnancy HF-HRV and heart rate as parallel mediators (Panel II, Figure 2) showed a unique indirect effect of early pregnancy depressive symptoms on gestational hypertension via late pregnancy HF-HRV $(b=$ $0.04, S E=.025 ; 95 \%$ CI $0.0038,0.1028)$ but not via late pregnancy heart rate $(b=-0.02, S E=$ $.017 ; 95 \%$ CI -.0587, 0.0103). Lower HF-HRV in late pregnancy was associated with higher odds of gestational hypertension $(\mathrm{OR}=0.13 ; 95 \% \mathrm{CI} 0.0178,0.7498)$. None of the covariates changed the indirect effect of early pregnancy depressed mood on gestational hypertension via late pregnancy HF-HRV.

\section{Discussion}

To our knowledge, this is the first study to examine whether cardiac vagal control may represent a possible mechanism linking depressive symptoms to gestational hypertension. Parasympathetic control of the heart is important to blood pressure regulation, such that prolonged reductions in vagal activation (indexed by less HF-HRV) can lead to increases in heart rate and cardiac output that may contribute to increases in blood pressure over time. In expectant mothers, a well-regulated system of parasympathetic cardiovascular control may be especially pertinent in light of the tremendous physiological demands required for adaptation to pregnancy. 
As such, reductions in parasympathetic cardiovascular control have the potential to contribute to pathological conditions such as gestational hypertension.

Within this context, this study investigated (1) the association of prenatal depressive symptoms with maternal heart rate and HF-HRV, and (2) the mediating influence of cardiac vagal control in the association between depressive symptoms and gestational hypertension. We observed that greater depressive symptoms were associated with relatively lower HF-HRV across pregnancy. In turn, there was an indirect effect of depressive symptoms on gestational hypertension via lower HF-HRV. After statistical adjustment for heart rate, the indirect effect was observed for late pregnancy HF-HRV, suggesting the incremental utility of measuring cardiac vagal control as a marker for gestational hypertension in the third trimester.

We did not directly measure sympathetic outflow to the heart, so the nature of any potential sympathetic-parasympathetic influences on cardiac function is not certain. We observed that HF-HRV accounted for roughly $40 \%$ of variance in heart rate, which suggests that higher heart rate is related to, but not purely determined by, lower cardiac vagal control—consistent with prior findings (Sandercock et al., 2008). Despite our inability to determine vagal versus sympathetic effects in the relationship between depressed mood in gestational hypertension, the present results supplement existing evidence of increased sympathetic activity in hypertensive disorders of pregnancy (Bosio et al., 1999; Greenwood et al., 2003) by suggesting that reduced vagal activity, as measured by HF-HRV, may also be relevant.

This study replicates and extends previous findings of associations between depression and cardiac vagal control by demonstrating that this association holds during pregnancy (Rottenberg, 2007). One previous study showed an association between depression during pregnancy and lower standard deviation of NN intervals (Shea et al., 2008); however, this 
measure of HRV is influenced by factors other than cardiac vagal activation (Thayer et al., 2008), making it difficult to interpret those results. There is no established cut-off for "problematic" levels of HF-HRV, including in pregnancy (Stein et al., 1999). However, to put the observed association in perspective, the significant beta-weight for depressive symptoms $(b=$ -0.02) indicates that a one-unit increase in an individual's EDS score is associated with a 0.02 unit decrease in log HF-HRV. Therefore, a 13-unit increase in an individual's EDS score (which represents the difference between a woman with no depressive symptoms, EDS $=0$, and a woman with clinically significant depressive symptoms, EDS $=13$ ) is associated with a 0.26 -unit decrease in $\log$ HF-HRV (which corresponds to approximately $3 / 4$ of a standard deviation). The sensitivity of HF-HRV to depressed mood in this sample is remarkable in light of considerable changes in cardiac autonomic modulation that occur with advancing gestation (de Weerth \& Buitelaar, 2005; Stein et al., 1999).

To interpret our observed association between cardiac vagal control and gestational hypertension, it is helpful to consider the odds of developing gestational hypertension in terms of a one standard deviation change in late pregnancy HF-HRV, given that a one unit change represents approximately 2.5 standard deviations. That is, our data show that a one standard deviation (i.e., $0.4 \ln \bullet \mathrm{ms}^{2}$ ) increase in late pregnancy HF-HRV is related to a $0.53 \log$ odds decrease in gestational hypertension. Using the $e^{\log \text { odds }}$ equation, this converts to an odds ratio of 0.45 , suggesting a $55 \%$ reduction in risk for gestational hypertension. Our findings are consistent with prospective research in non-pregnant adults suggesting lower cardiac vagal tone is associated with hypertension (Liao et al., 1996; Schroeder et al., 2003). However, the results of studies examining cardiac vagal control in gestational hypertension have been mixed, with some reporting no differences (Metsaars, Ganzevoort, Karemaker, Rang, \& Wolf, 2006), higher HF- 
HRV (Faber et al., 2004), and lower HF-HRV (Antonazzo, Cetin, Tarricone, Lombardi, \& Pardi, 2004; Yang, Chao, Kuo, Yin, \& Chen, 2000) compared to uncomplicated pregnancies. Overall, it is difficult to explain the equivocal findings because most research has been cross-sectional, laboratory-based, and has combined gestational hypertension with other etiologically distinct pregnancy complications.

These findings contribute new knowledge about potential mechanisms by which depressed mood might "get under the skin" of women to influence pregnancy outcomes. While it has been theorized that poor health behaviors help explain the relationships among psychological distress, vagal functioning, and cardiovascular health outcomes (Rottenberg, 2007; Thayer \& Lane, 2007; Whooley \& Wong, 2013), we observed an association between depressed mood and HRV after excluding women who reported behaviors such as current substance use and after statistically adjusting for indices of physical activity and pre-pregnancy BMI. To clarify the extent to which health behaviors account for the relation between depression and HF-HRV, future studies might consider the role of additional behaviors including diet, sleep, and adherence to prenatal care recommendations.

Our observation that a less responsive "vagal brake" (Porges, Doussard-Roosevelt, Portales, \& Greenspan, 1996), as indexed by relatively lower HF-HRV, was associated with gestational hypertension is in keeping with the important role of the autonomic nervous system in blood pressure regulation (Guyenet, 2006). The findings suggest that a component of the increased heart rate and cardiac output previously observed in gestational hypertension (Bosio et al., 1999) may at least partly reflect a reduction in parasympathetic activation of the heart. The question of when HF-HRV might influence the disease trajectory is complicated by the fact that we were unable to ascertain exact timing for the onset of gestational hypertension within study 
participants. We can only know that gestational hypertension occurred at some point after 20 weeks gestation, which was always after measurement of early pregnancy HF-HRV but could have been before, during, or after measurement of late pregnancy HF-HRV (see Figure 1).

It also remains uncertain whether cardiac vagal control might be implicated in other disorders of pregnancy (e.g., preeclampsia, HELLP syndrome), as we only assessed gestational hypertension in the present study. Interestingly, prior research demonstrates that preeclampsia (i.e., gestational hypertension with proteinuria) shows a different hemodynamic pattern than isolated gestational hypertension, with elevated cardiac output in early pregnancy but a crossover to low cardiac output and elevated peripheral resistance after disease onset (Bosio et al., 1999), suggesting autonomic nervous system activity may differ across hypertensive disorders of pregnancy. The exact means through which HF-HRV is associated with gestational hypertension remains to be determined, ideally through longitudinal studies that measure the exact timing of disease onset and that measure HF-HRV relative to more proximal influences on blood pressure such as vascular resistance and cardiac output.

It is also intriguing that depressed mood was associated with gestational hypertension only indirectly through HF-HRV. The lack of a direct effect in the present study suggests that psychobiological aspects of depressive symptoms may not directly influence control of blood pressure but instead, could exert their effects through intervening components of stress physiology. Complex, bidirectional pathways are likely, and might include variables such as rumination, neural regulatory processes, immune system activity, HPA-axis response, and health behaviors (Gerin et al., 2012). Additional investigation is needed to clarify these findings by incorporating other potential mediators. Further, certain symptoms associated with depression may be more "potent" than others in their influence on vagal functioning, such as rumination 
(Gerin et al., 2012; Pieper et al., 2007), suicidal ideation (Rottenberg, Wilhelm, Gross, \& Gotlib, 2002), and somatic symptoms (de Jonge, Mangano, \& Whooley, 2007). It would be beneficial for future research to deconstruct depressive symptoms to better understand which aspects show the strongest associations with vagus nerve function.

A notable strength of the present study is the incorporation of design elements that increase ecological validity, including wrist actigraphy and ambulatory monitoring of HRV, while accounting for important confounding influences. As a result, we avoided potential reactivity effects that may occur in lab-based recordings and gathered HRV data that reflect the everyday functioning of participants. Our prospective collection of multiple, ecologically valid samples of activity and HRV twice during pregnancy is consistent with the growing use of ecological momentary assessment to examine bio-behavioral models of prenatal psychological distress in expectant mothers (Entringer, Buss, Andersen, Chicz-DeMet, \& Wadhwa, 2011; Giesbrecht, Campbell, Letourneau, Kaplan, \& APrON Study Team, 2013; Giesbrecht, Campbell, Letourneau, Kooistra, \& Kaplan, 2012).

Despite the strengths of our work, several limitations deserve mention. Our use of retrospective chart review to assess gestational hypertension was not optimal. We were not able to standardize blood pressure measurement across the study sample, although we note that our data reflect clinical practice. For instance, clinical recommendations from the Society of Obstetricians and Gynaecologists of Canada suggest a diagnosis of gestational hypertension should be based on the average of at least two blood pressure measurements taken $\geq 15$ minutes apart using the same arm (Magee et al., 2014), but we cannot verify adherence to published recommendations by the physicians who diagnosed gestational hypertension in these participants. 
In addition, assessment of HRV in women's day-to-day lives may have led to increased variability in the data relative to controlled laboratory-based research. It is also possible that women with depression have a different physical activity pattern compared to those without given that anhedonia, fatigue, and psychomotor changes are common symptoms (American Psychiatric Association, 2013). We mitigated these limitations by measuring and accounting for physical activity and by excluding HRV data with excessive artifacts. However, these adjustments might not have been adequate to fully rule out potential confounding influences such as physical movement and cardiorespiratory fitness.

Concerns have been raised about the interpretation of HRV as an index of cardiac vagal control without adjusting for respiratory parameters, given that HRV can be affected by changes in the frequency and volume of respiration (Denver, Reed, \& Porges, 2007; Grossman, 1992; Grossman \& Taylor, 2007). There are similar concerns about between-subjects HRV comparisons because of individual differences in respiratory modulation of cardiac vagal control (Grossman \& Kollai, 1993; Kollai \& Mizsei, 1990). These associations are further complicated by observations that statistical adjustment for respiratory variables might obscure potential valid effects and non-linear relationships between respiratory and vagal influences on the heart (Denver et al., 2007; Thayer, Loerbroks, \& Sternberg, 2011). Our measurement of HRV was also limited in that heart rate was not sampled in the afternoon or during sleep. While HF-HRV is generally considered to be a valid index of cardiac vagal control and is frequently used in the literature to predict cardiovascular risk (Pomeranz et al., 1985; Thayer et al., 2010; Tsuji et al., 1994), it is limited because it is not a direct measure of parasympathetic influence on the heart.

While there are strong theoretical reasons to assume depressed mood could increase risk for gestational hypertension at least in part via changes in cardiac vagal control, causal ordering 
of the observed associations is not certain. The exact relation between cardiac vagal control and depression is unknown (Rottenberg, 2007). It could be that reduced vagal activity contributes to changes in depressive symptoms, as suggested by prior research showing that vagal nerve stimulation produces antidepressant effects (George et al., 2000; Schachter, 2004). There are also questions about whether cardiac vagal control is merely a marker versus a cause of underlying pathophysiology in gestational hypertension. We attempted to clarify causal pathways by measuring depressive symptoms prior to the development of gestational hypertension and prior to measurement of late pregnancy HF-HRV. Additional prospective studies are needed to develop causal claims.

Although the sample was adequate in size to detect some associations among depressive symptoms, HF-HRV, and gestational hypertension, other associations might have been underestimated due to limited statistical power. Power calculations based upon path analysis in the mediation models show that, with $80 \%$ power, an alpha of $5 \%$, and our observed $7 \%$ prevalence of gestational hypertension, the sample was adequate in size to detect a medium effect (corresponding to an odds ratio of 2.61 or its inverse 0.38 ) on gestational hypertension (Chen, Cohen, \& Chen, 2010; Hsieh, 1989). Associations of depressive symptoms with HF-HRV and gestational hypertension may have been further attenuated due to the relatively low severity of depression in this sample.

Also, our use of retrospective self-reported weight in the calculation of pre-pregnancy BMI is potentially biased. Roughly $1 / 5$ of individuals underestimate their weight (Cameron \& Evers, 1990; Jalkanen, Tuomilehto, Tanskanen, \& Puska, 1987; Jeffrey, 1996), although selfreported and actual weight tend to be highly correlated (i.e., 90\%) (Jeffrey, 1996; Stunkard \& Albaum, 1981). Our observation that higher pre-pregnancy BMI was associated with the 
tendency to develop gestational hypertension is in line with prior research (Nohr et al., 2008; Thadhani et al., 1999) and is concerning given the growing prevalence of overweight and obesity among women of childbearing age (Heslehurst, Rankin, Wilkinson, \& Summerbell, 2010; Kim, Dietz, England, Morrow, \& Callaghan, 2007). An additional limitation is that participants were predominantly non-Hispanic White, well-educated, married women with low levels of depressed mood. Our findings should be replicated in more diverse samples to determine whether these results generalize across the risk gradient. We were also not able to examine whether the results are similar for women with other hypertensive disorders including preeclampsia because of low incidence in the present study.

In summary, though it has been proposed that reduced cardiac vagal control may link depressive symptoms to hypertensive disorders (Liao et al., 1996; Shea et al., 2008), this is the first study to demonstrate this indirect effect in pregnant women. These preliminary results suggest changes in cardiac vagal control associated with maternal depressed mood are associated with gestational hypertension, either directly or as a vulnerability marker. Given that 15 to $25 \%$ of women diagnosed with gestational hypertension develop preeclampsia, greater etiologic understanding of gestational hypertension may help identify intervention targets to prevent preeclampsia and other severe complications such as fetal growth restriction, preterm delivery, placental abruption, eclampsia, and maternal and fetal death (Heazell et al., 2010). Further investigation is needed on potential interventions to improve obstetric and mental health outcomes in depressed pregnant women. Additional studies are also needed to explore the contribution of depressed mood and cardiac vagal control in the context of other pathogenic features of gestational hypertension (e.g., sympathetic nervous system activity, placental damage, dysregulated cell death, low lying placenta; Heazell et al., 2010); to ascertain the causal 
pathways linking depressed mood, cardiac vagal control, and hypertension during pregnancy; to evaluate whether vagal functioning can be modified as a result of treating maternal depression; and to determine whether such modification in HRV can prevent gestational hypertension. 


\section{Disclosures Statement}

The authors report no conflicts of interest. This research was supported in part by grants from Alberta Innovates Health Solutions, the Canadian Institutes of Health Research; the Alberta Centre for Child, Family and Community Research; and the Alberta Children's Hospital Research Institute. The sources of funding had no role in the study design, in the collection, analysis or interpretation of data; in writing the manuscript; or in the decision to submit the manuscript for publication. 


\section{Acknowledgements}

The authors gratefully acknowledge the participants of the Alberta Pregnancy Outcomes and Nutrition (APrON) study and the support of the APrON Study Team, whose individual members are B. J. Kaplan, C. J. Field, D. Dewey, R. C. Bell, F. P. Bernier, M. Cantell, L. M. Casey, M. Eliasziw, A. Farmer, L. Gagnon, G. F. Giesbrecht, L. Goonewardene, D. W. Johnston, L. Kooistra, N. Letourneau, M. O’Beirne, D. P. Manca, J. W. Martin, L. J. McCargar, V. J. Pop, and N. Singhal. The authors also thank T. O’Neill for his assistance with data analysis, as well as L. van den Hadelkamp, J. Thomas, and K. Lidgren for their assistance with data processing. 


\section{References}

Alder, J., Fink, N., Bitzer, J., Hösli, I., \& Holzgreve, W. (2007). Depression and anxiety during pregnancy: a risk factor for obstetric, fetal and neonatal outcome? A critical review of the literature. The Journal of Maternal-Fetal and Neonatal Medicine, 20(3), 189-209. doi: $10.1080 / 14767050701209560$

American Psychiatric Association. (2013). Diagnostic and Statistical Manual of Mental Disorders (5th ed.). Arlington, VA: American Psychiatric Publishing.

Antonazzo, P., Cetin, I., Tarricone, D., Lombardi, F., \& Pardi, G. (2004). Cardiac autonomic modulation in normal, high-risk, and in vitro fertilization pregnancies during the first trimester. American Journal of Obstetrics and Gynecology, 190(1), 199-205. doi:10.1016/S0002-9378(03)00896-2

Beevers, G., Lip, G. Y., \& O’Brien, E. (2001). ABC of hypertension: The pathophysiology of hypertension. BMJ (Clinical Research Ed.), 322(7291), 912-916. doi:10.1136/bmj.322.7291.912

Bernardi, L., Valle, F., Coco, M., Calciati, A., \& Sleight, P. (1996). Physical activity influences heart rate variability and very-low-frequency components in Holter electrocardiograms. Cardiovascular Research, 32(2), 234-237.

Berntson, G. G., Bigger, J. T. J., Eckberg, D. L., Grossman, P., Kaufmann, P. G., Malik, M., ... van der Molen, M. W. (1997). Heart rate variability: Origins, methods, and interpretative caveats. Psychophysiology, 34, 623-648.

Berntson, G. G., Cacioppo, J. T., \& Quigley, K. S. (1991). Autonomic determinism: the modes of autonomic control, the doctrine of autonomic space, and the laws of autonomic constraint. Psychological Review, 98(4), 459-87. 
Berntson, G. G., Quigley, K. S., Jang, J. F., \& Boysen, S. T. (1990). An approach to artifact identification: Application to heart period data. Psychophysiology, 27(5), 586-598.

Blackwell, T., Redline, S., Ancoli-Israel, S., Schneider, J. L., Surovec, S., Johnson, N. L., ... Study of Osteoporotic Fractures Research Group. (2008). Comparison of sleep parameters from actigraphy and polysomnography in older women: The SOF study. Sleep, 31(2), 28391.

Bosio, P., McKenna, P., Conroy, R., \& O’Herlihy, C. (1999). Maternal central hemodynamics in hypertensive disorders of pregnancy. Obstet Gynecol, 94, 978-984.

Cameron, R., \& Evers, S. (1990). Self-reported issues in obesity and weight management: state of the art and future directions. Behav Assess, 12, 91-106.

Carney, R. M., Freedland, K. E., Miller, G. E., \& Jaffe, A. S. (2002). Depression as a risk factor for cardiac mortality and morbidity: a review of potential mechanisms. Journal of Psychosomatic Research, 53, 897-902.

Chalmers, J. A., Quintana, D. S., Abbott, M. J.-A., \& Kemp, A. H. (2014). Anxiety disorders are associated with reduced heart rate variability: A meta-analysis. Frontiers in Psychiatry, 5(80), 1-11. doi:10.3389/fpsyt.2014.00080

Chen, H., Cohen, P., \& Chen, S. (2010). How big is a big odds ratio? Interpreting the magnitudes of odds ratios in epidemiological studies. Communications in Statistics - Simulation and Computation, 39(4), 860-864. doi:10.1080/03610911003650383

Clark, L. A., Watson, D., \& Mineka, S. (1994). Temperament, personality, and the mood and anxiety disorders. Special issue: personality and psychopathology. Journal of Abnormal Psychology, 103, 103-116. 
Cox, J. L., Holden, J. M., \& Sagovsky, R. (1987). Detection of postnatal depression. Development of the 10-item Edinburgh Postnatal Depression Scale. The British Journal of Psychiatry, 150(6), 782-786. doi:10.1192/bjp.150.6.782

Cripe, S. M., Frederick, I. O., Qiu, C., \& Williams, M. A. (2011). Risk of preterm delivery and hypertensive disorders of pregnancy in relation to maternal comorbid mood and migraine disorders during pregnancy. Paediatric and Perinatal Epidemiology, 25(2), 116-123. doi:10.1111/j.1365-3016.2010.01182.x.Risk

De Jonge, P., Mangano, D., \& Whooley, M. A. (2007). Differential association of cognitive and somatic depressive symptoms with heart rate variability in patients with stable coronary heart disease: findings from the Heart and Soul study. Psychosomatic Medicine, 69(8), 735739. doi:10.1097/PSY.0b013e31815743ca.Differential

De Paco, C., Kametas, N., Rencoret, G., Strobl, I., \& Nicolaides, K. H. (2008). Maternal cardiac output between 11 and 13 weeks of gestation in the prediction of preeclampsia and small for gestational age. Obstetrics and Gynecology, 111(2 Pt 1), 292-300.

doi:10.1097/01.AOG.0000298622.22494.0c

De Weerth, C., \& Buitelaar, J. K. (2005). Physiological stress reactivity in human pregnancy--a review. Neuroscience and Biobehavioral Reviews, 29(2), 295-312. doi:10.1016/j.neubiorev.2004.10.005

Denver, J. W., Reed, S. F., \& Porges, S. W. (2007). Methodological issues in the quantification of respiratory sinus arrhythmia. Biol Psychol, 74(2), 286-294. doi:10.1016/j.biotechadv.2011.08.021.Secreted 
Dunkel-Schetter, C. (2011). Psychological science on pregnancy: stress processes, biopsychosocial models, and emerging research issues. Annual Review of Psychology, 62, 531-558. doi:10.1146/annurev.psych.031809.130727

Dunkel-Schetter, C., \& Tanner, L. (2012). Anxiety, depression and stress in pregnancy: implications for mothers, children, research, and practice. Curr Opin Psychiatry., 25(2), 141-148. doi:10.1097/YCO.0b013e3283503680.Anxiety

Efron, B., \& Tibshirani, R. (1993). An introduction to the bootstrap. Boca Raton, FL: CRC Press LLC.

Entringer, S., Buss, C., Andersen, J., Chicz-DeMet, A., \& Wadhwa, P. D. (2011). Ecological momentary assessment of maternal cortisol profiles over a multiple-day period predict the length of human gestation. Psychosomatic Medicine, 73(6), 469-474.

doi:10.1097/PSY.0b013e31821 fbf9a.Ecological

Faber, R., Baumert, M., Stepan, H., Wessel, N., Voss, A., \& Walther, T. (2004). Baroreflex sensitivity, heart rate, and blood pressure variability in hypertensive pregnancy disorders. Journal of Human Hypertension, 18(10), 707-12. doi:10.1038/sj.jhh.1001730

Friedman, B. H., \& Thayer, J. F. (1998). Autonomic balance revisited: Panic anxiety and heart rate variability. Journal of Psychosomatic Research, 44(1), 133-151.

Fuchs, F. D., Chambless, L. E., Whelton, P. K., Nieto, F. J., \& Heiss, G. (2001). Alcohol consumption and the incidence of hypertension: The Atherosclerosis Risk in Communities Study. Hypertension, 37(5), 1242-1250. doi:10.1161/01.HYP.37.5.1242

Garcia, A. W., Langenthal, C. R., Angulo-Barroso, R. M., \& Gross, M. M. (2004). A comparison of accelerometers for predicting energy expenditure and vertical ground reaction force in 
school-age children. Measurement in Physical Education and Exercise Science, 8(3), 119144. doi:10.1207/s15327841mpee0803_1

George, M. S., Sackeim, H. A., Marangell, L. B., Husain, M. M., Nahas, Z., Lisanby, S. H., ... Rush, A. J. (2000). Vagus nerve stimulation: a potential therapy for resistant depression? Psychiatric Clinics of North America, 23(4), 757-783.

Gerin, W., Zawadzki, M. J., Brosschot, J. F., Thayer, J. F., Christenfeld, N. J. S., Campbell, T. S., \& Smyth, J. M. (2012). Rumination as a mediator of chronic stress effects on hypertension: a causal model. International Journal of Hypertension, 2012, 1-9. doi: $10.1155 / 2012 / 453465$

Giesbrecht, G. F., Campbell, T., Letourneau, N., Kaplan, B. J., \& APrON Study Team. (2013). Advancing gestation does not attenuate biobehavioural coherence between psychological distress and cortisol. Biological Psychology, 93(1), 45-51.

doi:10.1016/j.biopsycho.2013.01.019

Giesbrecht, G. F., Campbell, T., Letourneau, N., Kooistra, L., \& Kaplan, B. (2012). Psychological distress and salivary cortisol covary within persons during pregnancy. Psychoneuroendocrinology, 37(2), 270-9. doi:10.1016/j.psyneuen.2011.06.011

Greenwood, J. P., Scott, E. M., Walker, J. J., Stoker, J. B., \& Mary, D. A. S. G. (2003). The magnitude of sympathetic hyperactivity in pregnancy-induced hypertension and preeclampsia. American Journal of Hypertension, 16, 194-199.

Grossman, P. (1992). Respiratory and cardiac rhythms as windows to central and autonomic biobehvioral regulation. Biological Psychology, 34(2), 131-161. 
Grossman, P., \& Kollai, M. (1993). Respiratory sinus arrhythmia, cardiac vagal tone, and respiration: within- and between-individual relations. Psychophysiology, 30(5), 486-495. doi:10.1111/j.1469-8986.1993.tb02072.x

Grossman, P., \& Taylor, E. W. (2007). Toward understanding respiratory sinus arrhythmia: Relations to cardiac vagal tone, evolution and biobehavioral functions. Biological Psychology, 74(2), 263-285. doi:10.1016/j.biopsycho.2005.11.014

Guyenet, P. G. (2006). The sympathetic control of blood pressure. Nature Reviews Neuroscience, 7(5), 335-346. doi:10.1038/nrn1902

Hayes, A. F. (2013). Introduction to mediation, moderation, and conditional process analysis: A regression based approach. New York, NY: The Guilford Press.

Heazell, A., Norwitz, E. R., Kenny, Louise, C., \& Baker, P. N. (2010). Hypertension in Pregnancy. New York, NY: Cambridge University Press.

Heslehurst, N., Rankin, J., Wilkinson, J. R., \& Summerbell, C. D. (2010). A nationally representative study of maternal obesity in England, UK: trends in incidence and demographic inequalities in 619323 births, 1989-2007. International Journal of Obesity, 34(3), 420-428. doi:10.1038/ijo.2009.250

Horsten, M., Ericson, M., Perski, A., Wamala, S. P., Schenck-Gustafsson, K., \& Orth-Gomér, K. (1999). Psychosocial factors and heart rate variability in healthy women. Psychosomatic Medicine, 61(1), 49-57.

Hosmer, D. W. J., Lemeshow, S., \& Sturdivant, R. X. (2013). Applied Logistic Regression (3rd ed.). John Wiley \& Sons.

Hsieh, F. Y. (1989). Sample size tables for logistic regression. Statistics in Medicine, 8(7), 795802. doi:10.1002/sim.4780080704 
Jalkanen, L., Tuomilehto, J., Tanskanen, A., \& Puska, P. (1987). Accuracy of self-reported body weight compared to measured body weight. A population survey. Scand J Soc Med, 15, 191-198.

Jean-Louis, G., Kripke, D. F., Mason, W. J., Elliott, J. A., \& Youngstedt, S. D. (2001). Sleep estimation from wrist movement quantified by different actigraphic modalities. Journal of Neuroscience Methods, 105(2), 185-191.

Jeffrey, R. (1996). Bias in reported body weight as a function of education, occupation, health and weight concern. Addict Behav, 21, 217-222.

Kang, M. G., Koh, S. B., Cha, B. S., Park, J. K., Woo, J. ., \& Chang, S. J. (2004). Association between job stress on heart rate variability and metabolic syndrome in shipyard male workers. Yonsei Medical Journal, 45(5), 838-846.

Kim, S. Y., Dietz, P. M., England, L., Morrow, B., \& Callaghan, W. M. (2007). Trends in prepregnancy obesity in nine states, 1993-2003. Obesity, 15(4), 986-993. doi:10.1038/oby.2007.621

Klinkenberg, A. V, Nater, U. M., Nierop, A., Bratsikas, A., Zimmermann, R., \& Ehlert, U. (2009). Heart rate variability changes in pregnant and non-pregnant women during standardized psychosocial stress. Acta Obstetricia et Gynecologica, 88, 77-82. doi:10.1080/00016340802566762

Kollai, M., \& Mizsei, G. (1990). Respiratory sinus arrhythmia is a limited measure of cardiac parasympathetic control in man. The Journal of Physiology, 424, 329-342.

Kreibig, S. D. (2010). Autonomic nervous system activity in emotion: a review. Biological Psychology, 84(3), 394-421. doi:10.1016/j.biopsycho.2010.03.010 
Lee, A. M., Lam, S. K., Lau, S. M. S. M., Chong, C. S. Y., Chui, H. W., \& Fong, D. Y. T. (2007). Prevalence, course, and risk factors for antenatal anxiety and depression. Obstetrics and Gynecology, 110(5), 1102-1112.

Leeners, B., Neumaier-Wagner, P., Kuse, S., Stiller, R., \& Rath, W. (2007). Emotional stress and the risk to develop hypertensive diseases in pregnancy. Hypertension in Pregnancy, 26(2), 211-26. doi:10.1080/10641950701274870

Liao, D., Cai, J., Barnes, R. W., Tyroler, H. A., Rautaharju, P., Hohne, I., \& Heiss, G. (1996). Association of cardiac automatic function and the development of hypertension: The ARIC Study. American Journal of Hypertension, 9(12), 1147-1156.

Magee, L. A., Pels, A., Helewa, M., Rey, E., von Dadelszen, P., \& Hypertension Guideline Committee. (2014). Diagnosis, evaluation, and management of the hypertensive disorders of pregnancy: Executive summary. Journal of Obstetrics and Gynaecology Canada, 36(5), $416-438$.

Matthey, S. (2004). Calculating clinically significant change in postnatal depression studies using the Edinburgh Postnatal Depression Scale. Journal of Affective Disorders, 78(3), 269272. doi:10.1016/S0165-0327(02)00313-0

McEwen, B. S., \& Seeman, T. (2006). Protective and damaging effects of mediators of stress elaborating and testing the concepts of allostasis and allostatic load. Annals of the New York Academy of Sciences, 896(1), 30-47.

Meng, L., Chen, D., Yang, Y., Zheng, Y., \& Hui, R. (2012). Depression increases the risk of hypertension incidence: a meta-analysis of prospective cohort studies. Journal of Hypertension, 30(5), 842-851. doi:10.1097/HJH.0b013e32835080b7 
Metsaars, W. P., Ganzevoort, W., Karemaker, J. M., Rang, S., \& Wolf, H. (2006). Increased sympathetic activity present in early hypertensive pregnancy is not lowered by plasma volume expansion. Hypertension in Pregnancy, 25(3), 143-57. doi:10.1080/10641950600912927

Meyers, L. S., Gamst, G., \& Guarino, A. J. (2013). Applied Multivariate Research: Design and Interpretation. Thousand Oaks, CA: Sage Publications.

Miller, G., Chen, E., \& Cole, S. W. (2009). Health psychology: developing biologically plausible models linking the social world and physical health. Annual Review of Psychology, 60, 501524. doi:10.1146/annurev.psych.60.110707.163551

Nast, I., Bolten, M., Meinlschmidt, G., \& Hellhammer, D. H. (2013). How to measure prenatal stress? A systematic review of psychometric instruments to assess psychosocial stress during pregnancy. Paediatric and Perinatal Epidemiology, 27(4), 313-22. doi:10.1111/ppe.12051

Nohr, E. A., Vaeth, M., Baker, J. L., Sørensen, T. I., Olsen, J., \& Rasmussen, K. M. (2008). Combined associations of prepregnancy body mass index and gestational weight gain with the outcome of pregnancy. American Journal of Clinical Nutrition, 342, 10-15. doi:10.3945/ajen.2008.26939.Combined

Pal, G. K., Shyma, P., Habeebullah, S., Pal, P., Nanda, N., \& Shyjus, P. (2011). Vagal withdrawal and sympathetic overactivity contribute to the genesis of early-onset pregnancyinduced hypertension. International Journal of Hypertension, 2011, 1-9. doi: $10.4061 / 2011 / 361417$ 
Patterson, S. M., Krantz, D. S., Montgomery, L. C., Deuster, P. A., Hedges, S. M., \& Nebel, L. E. (1993). Automated physical activity monitoring: validation and comparison with physiological and self-report measures. Psychophysiology, 30(3), 296-305.

Pieper, S., Brosschot, J. F., van der Leeden, R., \& Thayer, J. F. (2007). Cardiac effects of momentary assessed worry episodes and stressful events. Psychosomatic Medicine, 69(9), 901-909. doi:10.1097/PSY.0b013e31815a9230

Pomeranz, B., Macaulay, R. J., Caudill, M. A., Kutz, I., Adam, D., Gordon, D., ... Cohen, R. J. (1985). Assessment of autonomic function in humans by heart rate spectral analysis. The American Journal of Physiology, 248, H151-3.

Porges, S. W., Doussard-Roosevelt, J. A., Portales, A. L., \& Greenspan, S. I. (1996). Infant regulation of the vagal "brake" predicts child behavior problems: a psychobiological model of social behavior. Developmental Psychobiology, 29(8), 697-712. doi:10.1002/(SICI)10982302(199612)29:8\&lt;697::AID-DEV5\&gt;3.0.CO;2-O

Purves, D., Augustine, G. J., Fitzpatrick, D., Katz, L. C., LaMantia, A., McNamara, J. O., \& Williams, S. M. (2001). Autonomic regulation of cardiovascular function. In Neuroscience (2nd ed.). Sunderland: Sinauer Associates.

Rang, S., Wolf, H., Montfrans, G. a, \& Karemaker, J. M. (2002). Non-invasive assessment of autonomic cardiovascular control in normal human pregnancy and pregnancy-associated hypertensive disorders: a review. Journal of Hypertension, 20(11), 2111-2119.

Rini, C. K., Dunkel-Schetter, C., Hobel, C. J., Glynn, L. M., \& Sandman, C. A. (2006). Effective social support: Antecedents and consequences of partner support during pregnancy. Personal Relationships, 13(2), 207-229. doi:10.1111/j.1475-6811.2006.00114.x 
Rini, C. K., Dunkel-Schetter, C., Wadhwa, P. D., \& Sandman, C. A. (1999). Psychological adaptation and birth outcomes: The role of personal resources, stress, and sociocultural context in pregnancy. Health Psychology, 18(4), 333-345.

Rottenberg, J. (2007). Cardiac vagal control in depression: a critical analysis. Biological Psychology, 74, 200-211. doi:10.1016/j.biopsycho.2005.08.010

Rottenberg, J., Wilhelm, F. H., Gross, J. J., \& Gotlib, I. H. (2002). Respiratory sinus arrhythmia as a predictor of outcome in major depressive disorder. Journal of Affective Disorders, 71(1-3), 265-272. doi:10.1016/S0165-0327(01)00406-2

Sandercock, G., Gladwell, V., Dawson, S., Nunan, D., Brodie, D., \& Beneke, R. (2008). Association between RR interval and high-frequency heart rate variability acquired during short-term, resting recordings with free and paced breathing. Physiological Measurement, $29,795-802$.

Schachter, S. C. (2004). Vagus nerve stimulation: mood and cognitive effects. Epilepsy \& Behavior, 5, 56-59. doi:10.1016/j.yebeh.2003.11.007

Schroeder, E. B., Liao, D., Chambless, L. E., Prineas, R. J., Evans, G. W., \& Heiss, G. (2003). Hypertension, blood pressure, and heart rate variability: The Atherosclerosis Risk in Communities (ARIC) Study. Hypertension, 42(6), 1106-1111. doi:10.1161/01.HYP.0000100444.71069.73

Shakeel, N., Eberhard-Gran, M., Sletner, L., Slinning, K., Martinsen, E. W., Holme, I., \& Jenum, A. K. (2015). A prospective cohort study of depression in pregnancy, prevalence and risk factors in a multi-ethnic population. BMC Pregnancy and Childbirth, 15(5), 1-11. doi:10.1186/s12884-014-0420-0 
Shea, A. K., Kamath, M. V, Fleming, A., Streiner, D. L., Redmond, K., \& Steiner, M. (2008). The effect of depression on heart rate variability during pregnancy. A naturalistic study. Clinical Autonomic Research: Official Journal of the Clinical Autonomic Research Society, 18(4), 203-12. doi:10.1007/s10286-008-0480-1

Sibai, B. M. (2003). Diagnosis and management of gestational hypertension and preeclampsia. Obstetrics \& Gynecology, 102(1), 181-192. doi:10.1016/S0029-7844(03)00475-7

Staneva, A., Bogossian, F., Pritchard, M., \& Wittkowski, A. (2015). The effects of maternal depression, anxiety, and perceived stress during pregnancy on preterm birth: A systematic review. Women and Birth. doi:10.1016/j.wombi.2015.02.003

Stein, P. K., Hagley, M. T., Cole, P. L., Domitrovich, P. P., Kleiger, R. E., \& Rottman, J. N. (1999). Changes in 24-hour heart rate variability during normal pregnancy. American Journal of Obstetrics and Gynecology, 180(4), 978-985.

Stunkard, A., \& Albaum, J. (1981). The accuracy of self-reported weights. Clin Nutr, 34, 15931599.

Task Force. (1996). Heart rate variability: Standards of measurement, interpretation, and clinical use. European Heart Journal, 17, 354-381.

Thadhani, R., Stampfer, M. J., Hunter, D. J. ., Manson, J. E., Solomon, C. G., \& Curhan, G. C. (1999). High body mass index and hypercholesterolemia: risk of hypertensive disorders of pregnancy. Obstetrics \& Gynecology, 94(4), 543-550.

Thayer, J. F., Friedman, B. H., \& Borkovec, T. D. (1996). Autonomic characteristics of generalized anxiety disorder and worry. Biological Psychiatry, 39(4), 255-266. doi:10.1016/0006-3223(95)00136-0 
Thayer, J. F., Hansen, A. L., \& Johnsen, B. J. (2008). Noninvasive assessment of autonomic influences on the heart. In L. J. Luecken \& L. C. Gallo (Eds.), Handbook of Physiological Research Methods in Health Psychology (pp. 183-209). Thousand Oaks, CA: Sage Publications.

Thayer, J. F., \& Lane, R. D. (2007). The role of vagal function in the risk for cardiovascular disease and mortality. Biological Psychology, 74(2), 224-42.

doi:10.1016/j.biopsycho.2005.11.013

Thayer, J. F., Loerbroks, A., \& Sternberg, E. M. (2011). Inflammation and cardiorespiratory control: The role of the vagus nerve. Respiratory Physiology and Neurobiology, 178(3), 387-394. doi:10.1016/j.resp.2011.05.016

Thayer, J. F., Yamamoto, S. S., \& Brosschot, J. F. (2010). The relationship of autonomic imbalance, heart rate variability and cardiovascular disease risk factors. International Journal of Cardiology, 141, 122-131. doi:10.1016/j.ijcard.2009.09.543

Tsuji, H., Venditti, F. J. J., Manders, E. S., Evans, J. C., Larson, M. G., Feldman, C. L., \& Levy, D. (1994). Reduced heart rate variability and mortality risk in an elderly cohort. The Framingham Heart Study. Circulation, 90(2), 878-883. doi:10.1161/01.CIR.90.2.878

Whooley, M. A., \& Wong, J. M. (2013). Depression and cardiovascular disorders. Annual Review of Clinical Psychology, 9, 327-54. doi:10.1146/annurev-clinpsy-050212-185526

Yang, C. C. H., Chao, T., Kuo, T. B. J., Yin, C., \& Chen, H. I. (2000). Preeclamptic pregnancy is associated with increased sympathetic and decreased parasympathetic control of HR. American Journal of Physiology - Heart and Circulatory Physiology, 278, H1269-H1273. 
Zhang, S., Ding, Z., Liu, H., Chen, Z., Wu, J., Zhang, Y., \& Yu, Y. (2013). Association between mental stress and gestational hypertension/preeclampsia: A meta-analysis. Obstetrical \& Gynecological Survey, 68(12), 825-834. 
Table 1

Sample Characteristics $(n=287)$

\begin{tabular}{|c|c|c|c|c|c|c|}
\hline \multirow[b]{2}{*}{ Variable } & \multicolumn{3}{|c|}{$\begin{array}{l}\text { Women without Gestational } \\
\text { Hypertension }(n=268)\end{array}$} & \multicolumn{3}{|c|}{$\begin{array}{l}\text { Women with Gestational Hypertension } \\
\qquad(n=19)\end{array}$} \\
\hline & Mean & $S D$ & Range & Mean & $S D$ & Range \\
\hline \multicolumn{7}{|l|}{ Maternal characteristics } \\
\hline Maternal age (years) & 31.4 & 3.8 & $19.2-42.8$ & 30.7 & 3.7 & $24.5-37.0$ \\
\hline Pre-pregnancy BMI $\left(\mathrm{kg} / \mathrm{m}^{2}\right)$ & 24.6 & 4.9 & $15.7-44.8$ & 30.4 & 6.6 & $18.9-44.3$ \\
\hline Gestational age (weeks), Time 1 & 14.8 & 3.7 & $5.9-21.9$ & 16.3 & 1.9 & $13.1-20.0$ \\
\hline Gestational age (weeks), Time 2 & 32.4 & 1.0 & $27.1-36.7$ & 32.4 & 0.8 & $31.6-34.6$ \\
\hline \multicolumn{7}{|l|}{ Symptoms of depression and anxiety ${ }^{\mathrm{a}}$} \\
\hline Depressive symptoms (EDS), Time 1 & 5.7 & 4.2 & $0-20$ & 5.5 & 3.8 & $2-14$ \\
\hline Depressive symptoms (EDS), Time 2 & 5.3 & 4.1 & $0-23$ & 6.5 & 3.7 & $0-16$ \\
\hline Pregnancy-related anxiety (PRAS), Time 1 & 1.8 & 0.5 & $1.0-3.0$ & 1.9 & 0.4 & $1.2-2.7$ \\
\hline Pregnancy-related anxiety (PRAS), Time 2 & 1.7 & 0.4 & $1.0-3.2$ & 1.8 & 0.5 & $1.1-2.8$ \\
\hline \multicolumn{7}{|l|}{ Cardiac measures ${ }^{\mathrm{b}}$} \\
\hline Heart rate $(\mathrm{bpm})$, Time 1 & 87.2 & 9.7 & $63.3-118.6$ & 92.8 & 9.2 & $76.2-111.2$ \\
\hline Heart rate (bpm), Time 2 & 94.1 & 9.4 & $62.4-125.9$ & 94.6 & 8.0 & $79.7-112.9$ \\
\hline HF-HRV, $\ln \left(\mathrm{ms}^{2}\right)$, Time 1 & 2.4 & 0.3 & $1.5-3.6$ & 2.2 & 0.4 & $1.5-2.6$ \\
\hline HF-HRV, $\ln \left(\mathrm{ms}^{2}\right)$, Time 2 & 2.2 & 0.4 & $1.1-3.5$ & 1.9 & 0.4 & $1.0-2.6$ \\
\hline \multicolumn{7}{|l|}{ Actigraphy } \\
\hline Momentary activity score, Time 1 & 18793.3 & 5662.4 & $2426.1-36582.4$ & 19464.1 & 5827.2 & $8326.1-34899.2$ \\
\hline Momentary activity score, Time 2 & 18794.2 & 5895.4 & $4954.9-40457.7$ & 18267.4 & 4908.4 & $8592.1-26707.8$ \\
\hline Average activity score, Time 1 & 19327.7 & 3922.4 & $8459.5-29656.4$ & 20142.6 & 2917.9 & $16321.9-24969.3$ \\
\hline Average activity score, Time 2 & 18168.8 & 3827.0 & $7369.4-28917.2$ & 17545.9 & 3117.7 & $13054.7-24893.8$ \\
\hline Highest education attained & & $n$ & $\%$ & & $n$ & $\%$ \\
\hline Less than high school diploma & & 3 & 1.1 & & 0 & 0.0 \\
\hline Completed high school diploma & & 22 & 8.2 & & 4 & 21.1 \\
\hline Completed trade/technical school & & 49 & 18.3 & & 6 & 31.6 \\
\hline
\end{tabular}




\begin{tabular}{|c|c|c|c|c|}
\hline & $n$ & $\%$ & $n$ & $\%$ \\
\hline Completed undergraduate degree & 121 & 45.1 & 9 & 47.4 \\
\hline Completed post-graduate degree & 62 & 23.1 & 0 & 0.0 \\
\hline \multicolumn{5}{|l|}{ Annual household income } \\
\hline More than $\$ 100,000$ & 141 & 52.6 & 10 & 52.6 \\
\hline$\$ 70,000-\$ 99,999$ & 62 & 23.1 & 6 & 31.6 \\
\hline$\$ 40,000-\$ 69,000$ & 32 & 11.9 & 2 & 10.5 \\
\hline Less than $\$ 40,000$ & 22 & 8.2 & 1 & 5.3 \\
\hline \multicolumn{5}{|l|}{ Employment } \\
\hline Full-time & 208 & 77.6 & 18 & 94.7 \\
\hline Part-time & 52 & 19.4 & 1 & 5.3 \\
\hline \multicolumn{5}{|l|}{ Ethnicity } \\
\hline White & 205 & 76.5 & 17 & 89.5 \\
\hline Black & 3 & 1.1 & 0 & 0.0 \\
\hline Asian & 40 & 14.9 & 2 & 10.5 \\
\hline Hispanic & 11 & 4.1 & 0 & 0.0 \\
\hline Born in Canada & 194 & 72.4 & 17 & 89.5 \\
\hline \multicolumn{5}{|l|}{ Marital status } \\
\hline Married/common-law & 255 & 95.1 & 18 & 94.7 \\
\hline Single & 4 & 1.5 & 1 & 5.3 \\
\hline \multicolumn{5}{|l|}{ Gravida } \\
\hline 1 & 99 & 36.9 & 10 & 52.6 \\
\hline 2 & 93 & 34.7 & 4 & 21.1 \\
\hline 3 & 41 & 15.3 & 3 & 15.8 \\
\hline 4 & 21 & 7.8 & 2 & 10.5 \\
\hline 5 or greater & 6 & 2.2 & 0 & 0.0 \\
\hline Preeclampsia & 0 & 0.0 & 1 & 5.3 \\
\hline Use of antidepressants during pregnancy & 14 & 5.2 & & \\
\hline
\end{tabular}


Anxiety Scale. Gravida refers to number of pregnancies including the current pregnancy. Time $1=$ early pregnancy $(M=14.9$ weeks gestation), Time $2=$ late pregnancy $(M=32.4$ weeks gestation).

Table 2

Hierarchical Linear Models Examining Early Pregnancy Depressive Symptoms as a Predictor of Maternal High Frequency HRV and Heart Rate

\begin{tabular}{|c|c|c|c|c|c|c|}
\hline \multirow[b]{2}{*}{ Fixed Effects } & \multicolumn{3}{|c|}{ Model 1 (HF-HRV) } & \multicolumn{3}{|c|}{ Model 2 (Heart Rate) } \\
\hline & Coefficient (SE) & $t$ ratio & $d f$ & Coefficient (SE) & $t$ ratio & $d f$ \\
\hline Intercept & $3.23(0.202)$ & $15.98 * * *$ & 267 & $81.94(5.27)$ & $15.55 * * *$ & 267 \\
\hline \multicolumn{7}{|l|}{ Covariates } \\
\hline Pre-pregnancy BMI (kg/m²) & $-0.01(.005)$ & $-3.17 * *$ & 267 & $0.15(.126)$ & 1.25 & 267 \\
\hline Maternal age (years) & $-0.01(.005)$ & -1.23 & 267 & $-0.22(.135)$ & -1.64 & 267 \\
\hline Momentary activity (counts/epoch) ${ }^{\mathrm{a}}$ & $<-0.01(<.001)$ & $-9.81 * * *$ & 767 & $<-0.01(<.001)$ & $15.09 * * *$ & 767 \\
\hline Gestational age (weeks) & $-0.01(.001)$ & $-9.42 * * *$ & 728 & $0.39(.037)$ & $10.57 * * *$ & 728 \\
\hline \multicolumn{7}{|l|}{ Depressive symptoms } \\
\hline EDS & $-0.02(.005)$ & $-3.26 * *$ & 267 & $0.44(.115)$ & $3.81 * * *$ & 267 \\
\hline
\end{tabular}

Note. $\mathrm{BMI}=$ body mass index; EDS = Edinburgh Depression Scale, administered in early pregnancy; HF-HRV = high frequency heart rate variability; $\mathrm{SE}=$ standard error. All results represent final estimation of fixed effects with robust standard errors. HRV was tested for normality and required $\log$ transformation. $* p<.05, * * p<.01, * * * \leq .001$

aPerson mean centered. 
Table 3

Parallel Mediation Analyses Examining the Role of Maternal High Frequency Heart Rate Variability and Heart Rate in the Association between Prenatal Depressive Symptoms and Gestational Hypertension $(n=287)$

\begin{tabular}{|c|c|c|c|c|c|c|c|c|}
\hline \multirow[b]{2}{*}{ Effect } & \multicolumn{4}{|c|}{ Early Pregnancy HF-HRV and Heart Rate } & \multicolumn{4}{|c|}{$\begin{array}{c}\text { Late Pregnancy HF-HRV and Heart } \\
\text { Rate }\end{array}$} \\
\hline & $b$ & $S E$ & OR $(95 \% \mathrm{CI})$ & $R^{2}$ & $b$ & $S E$ & OR $(95 \% \mathrm{CI})$ & $R^{2}$ \\
\hline Direct effects on HF-HRV & & & & $.10^{* * *}$ & & & & $.12 * * *$ \\
\hline Maternal age (years) & -0.01 & .005 & & & $-0.01 *$ & .005 & & \\
\hline Pre-pregnancy BMI $\left(\mathrm{kg} / \mathrm{m}^{2}\right)$ & $-0.01 * * *$ & .004 & & & $-0.02 * * *$ & .004 & & \\
\hline $\begin{array}{l}\text { Momentary activity } \\
(\text { counts/epoch) })^{\mathrm{b}}\end{array}$ & $<0.01 * *$ & $<.001$ & & & $<0.01$ & $<.001$ & & \\
\hline EDS & $-0.01 *$ & .005 & & & $-0.02 * * *$ & .005 & & \\
\hline Direct effects on heart rate & & & & $.10 * * *$ & & & & $.07 * * *$ \\
\hline Maternal age (years) & -0.15 & .141 & & & -0.16 & .133 & & \\
\hline Pre-pregnancy BMI $\left(\mathrm{kg} / \mathrm{m}^{2}\right)$ & 0.17 & .112 & & & $0.21 *$ & .107 & & \\
\hline $\begin{array}{l}\text { Momentary activity } \\
(\text { counts/epoch) }\end{array}$ & $<0.01 * * *$ & $<.001$ & & & $<0.01 * *$ & $<.001$ & & \\
\hline EDS & $0.40 * *$ & .130 & & & $0.38 * *$ & .122 & & \\
\hline Direct effects on GH & & & & $.26^{\mathrm{a}}$ & & & & $.23^{\mathrm{a}}$ \\
\hline Maternal age (years) & -0.08 & .075 & $\begin{array}{c}0.92(0.80 \\
1.07)\end{array}$ & & -0.11 & .072 & $\begin{array}{c}0.90(0.78 \\
1.03)\end{array}$ & \\
\hline
\end{tabular}




\begin{tabular}{|c|c|c|c|c|c|c|}
\hline Pre-pregnancy BMI $\left(\mathrm{kg} / \mathrm{m}^{2}\right)$ & $0.19^{* * *}$ & .047 & $\begin{array}{c}1.20(1.10, \\
1.32)\end{array}$ & $0.18^{* * *}$ & .046 & $\begin{array}{c}1.20(1.10, \\
1.32)\end{array}$ \\
\hline $\begin{array}{l}\text { Momentary activity } \\
\text { (counts/epoch) }^{\mathrm{b}}\end{array}$ & $<0.01$ & $<.001$ & $\begin{array}{c}1.00(1.00, \\
1.00)\end{array}$ & $<0.01$ & $<.001$ & $\begin{array}{c}1.00(1.00, \\
1.00)\end{array}$ \\
\hline EDS & -0.05 & .070 & $\begin{array}{c}0.95(0.83, \\
1.09)\end{array}$ & -0.05 & .070 & $\begin{array}{c}0.95(0.83, \\
1.09)\end{array}$ \\
\hline$H F-H R V \ln \left(m s^{2}\right)$ & -1.32 & 1.04 & $\begin{array}{c}0.27(0.03, \\
2.05)\end{array}$ & $-2.02 *$ & 1.02 & $\begin{array}{c}0.13(0.02, \\
0.75)\end{array}$ \\
\hline Heart rate $(\mathrm{bpm})$ & 0.04 & .039 & $\begin{array}{c}1.04(0.96 \\
1.12)\end{array}$ & -0.05 & .043 & $\begin{array}{c}0.95(0.88, \\
1.04)\end{array}$ \\
\hline Indirect effects on $\mathrm{GH}$ & Effect $(95 \% \mathrm{CI})$ & $S E$ & & Effect $(95 \% \mathrm{CI})$ & $S E$ & \\
\hline $\mathrm{EDS} \rightarrow \mathrm{HF}-\mathrm{HRV} \rightarrow \mathrm{GH}$ & $\begin{array}{c}0.01(-.0053 \\
.0540)\end{array}$ & .014 & & $\begin{array}{c}0.04(.0038 \\
.1028)^{\dagger}\end{array}$ & .025 & \\
\hline EDS $\rightarrow$ Heart rate $\rightarrow \mathrm{GH}$ & $\begin{array}{c}0.02(-.0164, \\
.0666)\end{array}$ & .021 & & $\begin{array}{c}-0.02(-.0587 \\
.0103)\end{array}$ & .017 & \\
\hline
\end{tabular}

Note. $\mathrm{BMI}=$ body mass index; $\mathrm{CI}=$ confidence interval; $\mathrm{EDS}=$ Edinburgh Depression Scale, measured in early pregnancy; $\mathrm{GH}=$ gestational hypertension (coded dichotomously, $1=$ present, $0=$ absent); HF-HRV $=$ log-transformed high-frequency heart rate variability; $\mathrm{OR}=$ odds ratio; $\mathrm{SE}=$ standard error; bpm = beats per minute. All results represent final estimation of fixed effects with

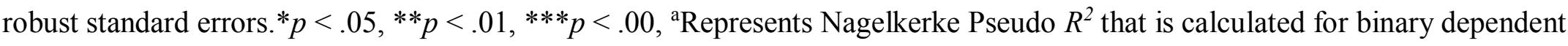
variables, for which no significance test is available. ${ }^{b}$ Person mean centered. "Significant indirect effect exists when confidence interval does not include 0 . 


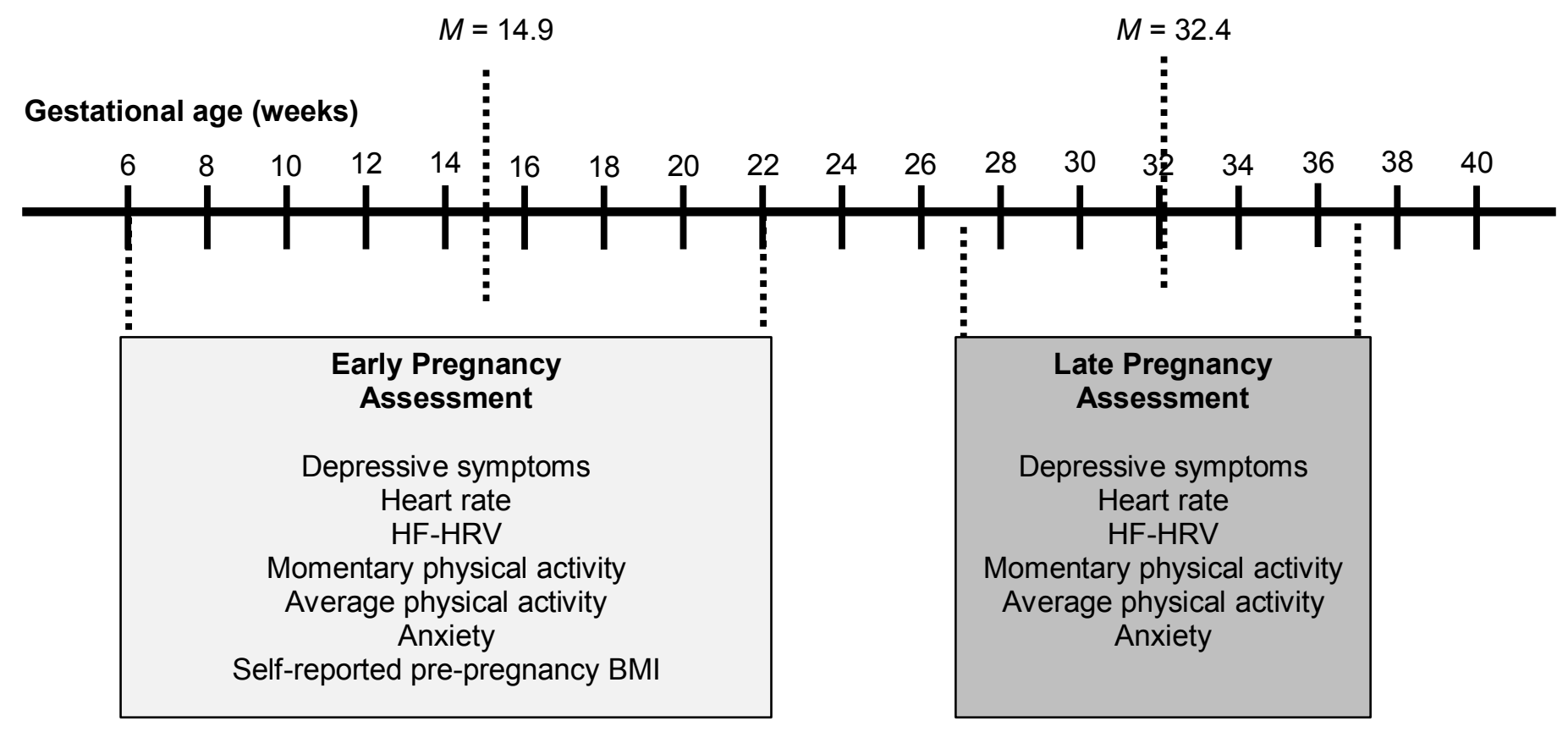

Figure 1. Assessment schedule for study variables. Gestational hypertension was dichotomous, determined by chart review following delivery. BMI = body mass index, HF-HRV = highfrequency heart rate variability. Gestational hypertension was diagnosed after 20 weeks gestation, and was determined by chart review following delivery. 
I

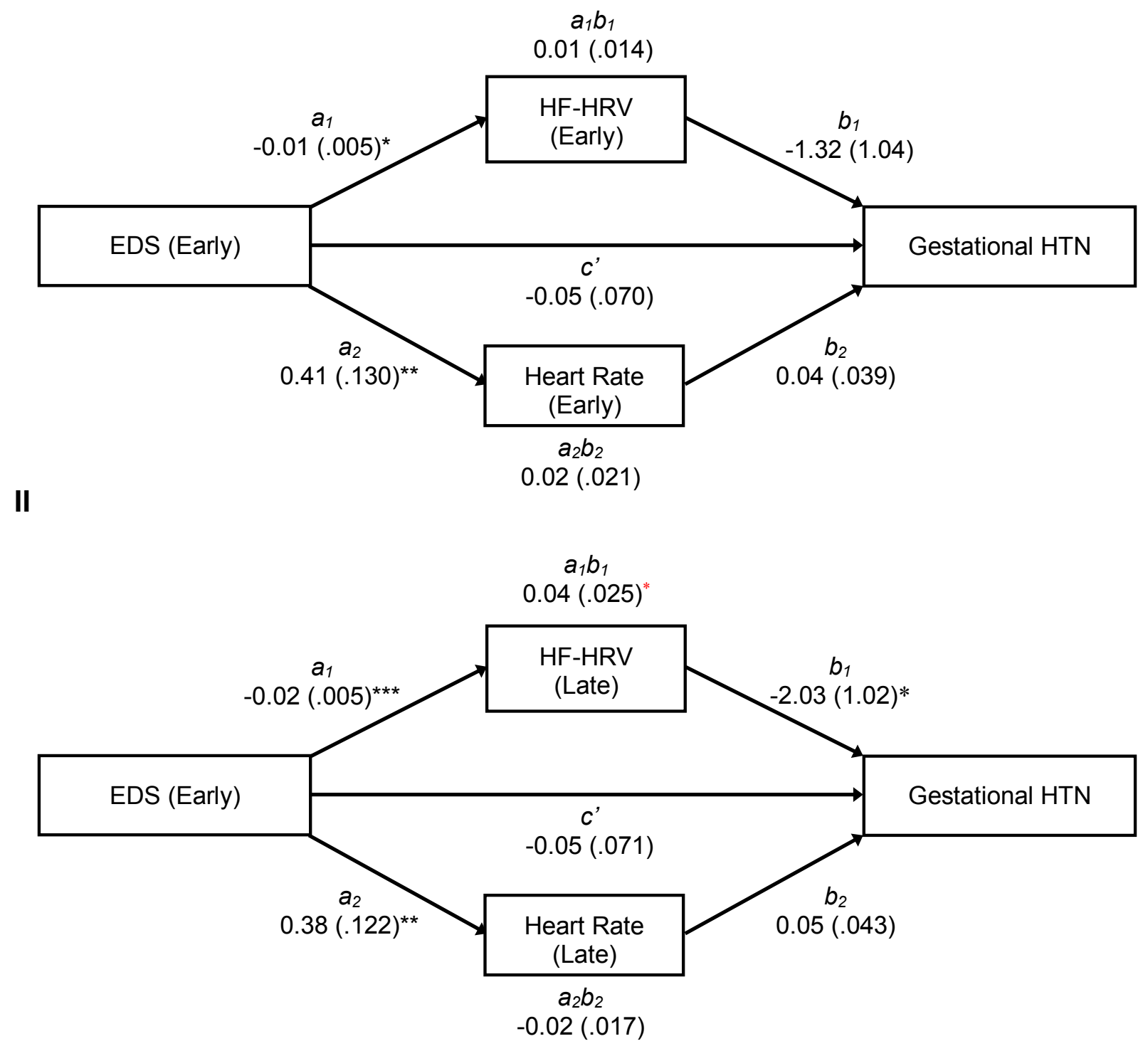

Figure 2. Early pregnancy depressive symptoms showed an indirect effect on gestational hypertension via lower late pregnancy HF-HRV (Panel II) but not via early pregnancy HF-HRV (Panel I), incremental to potential mediating effects of heart rate. Maternal age, pre-pregnancy body mass index, and physical activity were included as covariates in each model. Path values are unstandardized regression coefficients with SE in parentheses. Path $c$ ' represents the direct effect of depressive symptoms on gestational hypertension. Path $a_{1} b_{1}$ represents the indirect effect through changes in HF-HRV. Path $a_{2} b_{2}$ represents the indirect effect through changes in heart rate. EDS = Edinburgh Depression Scale; GH = gestational hypertension; HF-HRV = log-transformed high-frequency heart rate variability; HTN = hypertension.

${ }^{*} p<.05,{ }^{* *} p<.01, * * * p<.001$ 